\title{
Clinical Observation of Neoadjuvant Chemotherapy in High-Risk Stage II Colorectal Cancer: A Report of 24 Cases
}

\author{
Anxin Wang, Jinjun $\mathrm{Li}^{*}$ \\ Medical College, Wuhan University of Science and Technology, Wuhan, China \\ Email: *entry2003@126.com
}

How to cite this paper: Wang, A.X. and Li, J.J. (2017) Clinical Observation of Neoadjuvant Chemotherapy in High-Risk Stage II Colorectal Cancer: A Report of 24 Cases. Journal of Cancer Therapy, 8, 1169-1178. https://doi.org/10.4236/jct.2017.813100

Received: October 30, 2017

Accepted: December 11, 2017

Published: December 14, 2017

Copyright $\odot 2017$ by authors and Scientific Research Publishing Inc. This work is licensed under the Creative Commons Attribution International License (CC BY 4.0).

http://creativecommons.org/licenses/by/4.0/

\section{(c) (i) Open Access}

\begin{abstract}
Background: Neoadjuvant chemotherapy is the systemic chemotherapy before the start of local chemotherapy or radiotherapy. At present, neoadjuvant chemotherapy in the high-risk phase II (AJCC TNM stage II period, including T3N0M0 and T4N0M0) treatment of colon cancer reported less. The authors discussed the efficacy and safety of neoadjuvant chemotherapy in 24 patients with high-risk stage II colorectal cancer. Method: Forty-eight patients with stage II colorectal cancer were randomly divided into experimental group and control group, the postoperative complications, the side effects of chemotherapy drugs and the effect of chemotherapy drugs on the tumor were observed. The quality of life, survival time and tumor recurrence or metastasis was compared between the two groups. Result: 1) The incidence of complications in the neoadjuvant chemotherapy group was $20.8 \%$, the incidence of adjuvant chemotherapy was $25 \%$, and the overall complication rate was $22.9 \%$. P values were greater than 0.05.2) Through the comparison of the two groups of toxic and side effects, after SPASS software analysis, obtained P $>0.05$, the two groups were not statistically significant. 3) After 3 - 4 courses of preoperative chemotherapy, CR (complete remission) and $\mathrm{PD}$ (disease progression) patients were 0 cases, PR (partial remission) was 11 (45.8\%), NC/SD (no change) was $13(54.2 \%) .4)$ Through the comparison of the quality of life of the two groups of patients, from the function, symptoms and the overall situation to statistics, $P$ value $<0.05$, This comparison makes sense. 5) The 1-year survival rate was $100 \%(24 / 24)$ in the control group, with a 3-year survival rate of $92 \%$ $(22 / 24)$ and a 5 -year survival rate of $75 \%(9 / 12)$. All survival rates in the experimental group were $100 \%(24 / 24)$. 6) There were no recurrence or metastasis cases in the neoadjuvant chemotherapy group, and 2 cases of recurrence and 1 case of metastasis in the adjuvant chemotherapy group were compared with the recurrence or metastasis after tumor operation. The chi-square test is not
\end{abstract}


statistically significant. Conclusion: Neoadjuvant chemotherapy plays a role in patients with high-risk second-stage colon cancer, the experimental group benefited significantly, especially in quality of life and survival time. At the same time, neoadjuvant chemotherapy is also a safe treatment.

\section{Keywords}

Neoadjuvant Chemotherapy, Adjuvant Chemotherapy, Colon Cancer, Efficacy, Risk Factors

\section{Introduction}

Colon cancer is a common gastrointestinal cancer, and it is one of the fastest rising tumor incidences in China. Early tumor generally has no obvious symptoms, and the symptoms appear at the later period of the general stage. Although surgical resection of local lesions of colon cancer is positive, but if the occurrence of systemic spread or metastasis is still leading to death, for all patients, even if only identified local lesions, there is no evidence that cancer cells have occurred before surgery Blood transfer.

The discovery of adjuvant chemotherapy in the 1980s can reduce the risk of recurrence of colon cancer with high risk factors, for the first stage does not require postoperative adjuvant chemotherapy, stage III and IV colon cancer adjuvant chemotherapy after the standard treatment program, and for stage II colon cancer (T3N0M0, T4N0M0) in patients with adjuvant chemotherapy more, whether all the postoperative adjuvant chemotherapy has no conclusive conclusion. Because most of the patients with stage II colon cancer surgery alone achieved better results, but for some patients with stage II colon cancer, with small metastases or subclinical lesions, surgery cannot completely removed the risk of recurrence after surgery. It is generally accepted that patients with stage II risk factors can benefit from adjuvant chemotherapy, especially in patients with a low degree of differentiation [1].

At present, neoadjuvant chemotherapy in the high-risk phase II (AJCC TNM stage, II period, including T3NOM0 and T4NOM0) treatment of colon cancer reported less. The authors explore the role of neoadjuvant chemotherapy in patients with stage II colorectal cancer in order to play a guiding role in clinical treatment regimens. It is reported as follows.

\section{Materials and Methods}

\subsection{Clinical Data}

Retrospectively 2012-01-01 to 2014-07-01 in Xiaogan City Center for general surgery high-risk stage of colon cancer patients in 48 cases, including 27 males and 21 females, aged $45-70$ years, mean age 64.5 years old. Were divided into two groups, the experimental group was neoadjuvant chemotherapy group, the 
control group was adjuvant chemotherapy group. Tumor site: neoadjuvant chemotherapy group ascending colon 12 cases, transverse colon 4 cases, descending colon and sigmoid colon 8 cases; adjuvant chemotherapy group ascending colon 15 cases, transverse colon 3 cases, 6 cases of sigmoid colon. High risk factors: neoadjuvant chemotherapy in poorly differentiated carcinoma in 19 cases, moderate differentiation in 5 cases, 19 cases of T4, T3 in 5 cases, 3 cases of intestinal obstruction, 3 cases of vascular invasion, adjuvant chemotherapy group: 22 cases of poorly differentiated carcinoma, 3 cases of differentiation, 19 cases of T4, 5 cases of T3, 6 cases of intestinal obstruction, 2 cases of vascular invasion. See Table 1. Participants included in the study informed and signed informed consent.

\subsection{Criteria}

After admission, abdominal enhancement of CT prompted colonic tumors, preoperative clinical stage II; Preoperative electronic colonoscopy prompted colon cancer, Postoperative disease prompted colonic malignancy; all patients have high risk factors in one or more, such as T4 staging, the number of lymph nodes seized less than 12, poor degree of differentiation (mucinous adenocarcinoma signet ring cell carcinoma), combined with vascular ganglion nerve invasion, intestinal obstruction with intestinal perforation; No chemotherapy before admission, preoperative chemotherapy cycle 4 - 6 course of treatment; Liver and kidney heart and lung and bone marrow hematopoietic function can tolerate surgery and chemotherapy, do not merge other parts of the tumor, do not merge other diseases recently life-threatening; The patient agrees to participate in this

Table 1. Summary of patient basic information.

\begin{tabular}{lcc}
\hline \multicolumn{1}{c}{ Normal information } & Test group (cases) & Control group (cases) \\
\hline Gender & 14 & 13 \\
Male & 10 & 11 \\
Female & & 15 \\
Tumor site & 12 & 3 \\
Ascending colon & 4 & 6 \\
Transverse colon & 8 & \\
Descending colon and & & 21 \\
sigmoid colon & 19 & 3 \\
High risk factors & 5 & 19 \\
Poorly differentiated & 19 & 5 \\
Differentiation & 5 & 6 \\
T3 & 3 & 2 \\
T4 & 3 & \\
Intestinal obstruction & & \\
Vascular invasion & & \\
\hline
\end{tabular}


research topic. Exclude criteria: After admission, abdominal enhancement of CT prompted colonic tumors, preoperative stage I, III or IV; Before chemotherapy, chemotherapy, radiotherapy, immunotherapy or other treatment; Intraoperative exploration found that the tumor has been transferred; Combined with mental illness; The patient refused to participate in the study or refused to return; Can not tolerate surgery, with chemotherapy contraindications.

\subsection{Surgical Program}

All colon cancer surgery by the same chief physician in general anesthesia under the laparoscopic radical resection of colon cancer, intraoperative strict implementation of the principle of aseptic and tumor-free operation, Resection and resection methods follow the guidelines for surgical procedures, tumor mass after removal of the disease were sent to the disease.

\subsection{Chemotherapy}

Neoadjuvant chemotherapy group: Line oxaliplatin + capecitabine chemotherapy and symptomatic treatment, oxaliplatin use dose by body surface area calculated $130 \mathrm{mg} / \mathrm{m}^{2}$, intravenous infusion of $2-6$ hours, No major toxicity occurs once every 3 weeks after administration 1, capecitabine use dose of $1250 \mathrm{mg} / \mathrm{m}^{2}$, Twice a day orally, sooner or later each time, equal to the total daily dose of 2500 $\mathrm{mg} / \mathrm{m}^{2}$, Treatment for two weeks after the withdrawal of a week, three weeks for a treatment course. Chemotherapy 3 - 4 after treatment and then interval 3 - 4 weeks after laparoscopic resection of colon cancer, and then 3 - 4 courses of chemotherapy. Adjuvant chemotherapy group: No chemotherapy before surgery, Laparoscopic radical resection of colon cancer, 3 - 4 weeks after the operation of oxaliplatin + capecitabine chemotherapy and symptomatic treatment, Use and dose of concentric chemotherapy group, chemotherapy 6 - 8 course of treatment.

\subsection{Efficacy Evaluation and Follow-Up}

To observe the postoperative complications of the two groups of patients, the side effects of chemotherapy drugs and the role of chemotherapy drugs on the tumor, the quality of life, survival time and tumor recurrence or metastasis were compared between the two groups. The first three indicators are available during hospitalization, and the latter three indicators can be obtained by telephone, WeChat or outpatient review.

\subsection{Data Processing}

Using SPASS16.0 software package, Measurement data using the mean and standard deviation and $\mathrm{T}$ test, Counting data usage rate and the use of chisquare test, using the probability $\mathrm{P}$ value for statistical inference, $\mathrm{P}<0.05$ was statistically significant.

Among them, the quality of life assessment of patients use EORTC QLQ-C30 
(version 3) quality of life questionnaire. The calculated scores are normalized, with a higher score in the Functional Areas and General Health fields indicating better functional status and quality of life, and a higher score in the Symptoms field indicating more symptoms or problems (poorer quality of life).

\section{Result}

By comparing the intraoperative and postoperative complications of the two groups of patients, the experimental group (neoadjuvant chemotherapy group) postoperative pneumonia in 3 cases (12.5\%), intestinal obstruction in 1 case (4.1\%), anastomotic fistula in 1 case $(4.1 \%)$, vascular injury in 0 cases. The control group (adjuvant chemotherapy) pneumonia in 4 cases (16.7\%), intestinal obstruction in 1 case (4.1\%), anastomotic fistula in 0 cases, vascular injury in 1 case $(4.1 \%)$. The incidence of complications in the neoadjuvant chemotherapy group was $20.8 \%$, the incidence of adjuvant chemotherapy was $25 \%$, and the overall complication rate was $22.9 \%$. The chi-square test $\mathrm{P}$ value is greater than 0.05 , can be concluded that the experimental group no significant increase in postoperative complications, is relatively safe. See Table 2 .

By comparing the side effects of two groups of chemotherapy drugs, after analysis, $P>0.05$, showing no significant difference between the two groups of chemotherapy drugs side effects, neoadjuvant chemotherapy is a safe solution, patients can tolerate the treatment options. See Table 3

CR (complete remission) and PD (disease progression) were 0 patients with preoperative chemotherapy, and PR (partial remission) were compared with those of abdominal CT when abdominal CT was compared with preoperative examination. (45.8\%), NC/SD (no change) was 13 cases (54.2\%), it can be seen that preoperative chemotherapy has a significant effect on some patients. See Table 4.

Table 2. Comparison of intraoperative and postoperative complications.

\begin{tabular}{cccccc}
\hline Group & $\begin{array}{c}\text { Intraoperative } \\
\text { blood loss }(\mathrm{ml})\end{array}$ & Pneumonia & $\begin{array}{c}\text { Intestinal } \\
\text { obstruction }\end{array}$ & $\begin{array}{c}\text { Anastomotic } \\
\text { fistula }\end{array}$ & $\begin{array}{c}\text { Vascular } \\
\text { injury }\end{array}$ \\
\hline Test group $(\mathrm{n}=24)$ & $88.08 \pm 10.01$ & $3(12.5 \%)$ & $1(4.1 \%)$ & $1(4.1 \%)$ & 0 \\
control group $(\mathrm{n}=24)$ & $88.33 \pm 8.93$ & $4(16.7 \%)$ & $1(4.1 \%)$ & 0 & $1(4.1 \%)$ \\
$\mathrm{X}^{2}$ & & 2.0 & 2.0 & 2.0 & 2.0 \\
$\mathrm{P}$ & & $>0.05$ & $>0.05$ & $>0.05$ & $>0.05$ \\
\hline
\end{tabular}

Table 3. Comparison of side effects of chemotherapy toxicity.

\begin{tabular}{cccccc}
\hline Group & $\begin{array}{c}\text { Bone marrow } \\
\text { suppression }\end{array}$ & $\begin{array}{c}\text { Nausea } \\
\text { and vomiting }\end{array}$ & Hair loss & Neurotoxicity & $\begin{array}{c}\text { Liver } \\
\text { damage }\end{array}$ \\
\hline Testgroup $(\mathrm{n}=24)$ & $6(25 \%)$ & $19(79 \%)$ & $1(4.1 \%)$ & $2(8.3 \%)$ & $4(17 \%)$ \\
control group $(\mathrm{n}=24)$ & $7(29 \%)$ & $21(87.5 \%)$ & $0(0 \%)$ & $2(8.3 \%)$ & $6(25 \%)$ \\
$\mathrm{X}^{2}$ & 2.0 & 2.0 & 2.0 & 2.0 & 2.0 \\
$\mathrm{P}$ & $>0.05$ & $>0.05$ & $>0.05$ & $>0.05$ & $>0.05$ \\
\hline
\end{tabular}


Through the comparison of the quality of life of the two groups of patients (Using the EORTC QLQ C30 questionnaire), from the function, symptoms and overall situation to statistics, $\mathrm{P}$ value $<0.05$, compared with the significance of the experimental group of quality of life was significantly higher than the quality of life of the control group, it can be seen that patients with stage II colon cancer benefit from neoadjuvant chemotherapy. Neoadjuvant chemotherapy improves clinical efficacy. See Table 5.

Compare the survival time of the two groups of patients, the 1-year survival rate was $100 \%(24 / 24)$, the 1 -year survival rate was $100 \%(24 / 24)$, the 3 -year survival rate was $92 \%(22 / 24)$, the 5 -year survival rate For $75 \%(9 / 12)$, It can be seen that the survival rate of the experimental group was significantly higher in patients with stage II colorectal cancer. See Table 6.

Through the comparison of recurrence or metastasis after tumor surgery, Neoadjuvant chemotherapy group without recurrence or metastasis cases, adjuvant chemotherapy group can be seen in 2 cases of recurrence, 1 case of metastasis. The chi-square test, no statistical significance, may be due to too little sample, no obvious conclusion. See Table 7.

Table 4. Comparison of CT changes in the neoadjuvant chemotherapy group at admission and before surgery.

\begin{tabular}{ccccc}
\hline Group & CR & PR & NC/SD & PD \\
\hline test group $(\mathrm{n}=24)$ & 0 & 11 & 13 & 0 \\
rate & 0 & $45.8 \%$ & $54.2 \%$ & 0 \\
\hline
\end{tabular}

Table 5. Quality of life scores for both groups.

\begin{tabular}{cccc}
\hline Group & Function & Symptom & Overall health \\
\hline Test group $(\mathrm{n}=24)$ & $61.09 \pm 4.56$ & $42.66 \pm 9.59$ & $47.22 \pm 11.71$ \\
Control group $(\mathrm{n}=24)$ & $49.06 \pm 3.69$ & $55.77 \pm 7.75$ & $42.00 \pm 10.56$ \\
$\mathrm{~T}$ & 7.966 & 3.154 & 2.178 \\
$\mathrm{P}$ & $<0.05$ & $<0.05$ & $<0.05$ \\
\hline
\end{tabular}

Table 6. Comparison of survival time between the two groups of patients.

\begin{tabular}{cccc}
\hline Group & 1-year survival rate & 3-year survival rate & 5-year survival rate \\
\hline Test group $(\mathrm{n}=24)$ & $100 \%(24 / 24)$ & $100 \%(24 / 24)$ & $100 \%(10 / 10)$ \\
Control group $(\mathrm{n}=24)$ & $100 \%(24 / 24)$ & $92 \%(22 / 24)$ & $75 \%(9 / 12)$ \\
Average survival rate & $100 \%(48 / 48)$ & $95.8 \%(46 / 48)$ & $86.3 \%(19 / 22)$ \\
\hline
\end{tabular}

Table 7. Postoperative tumor recurrence or metastasis comparison.

\begin{tabular}{ccc}
\hline Group & Relapse & Transfer \\
\hline Test group $(\mathrm{n}=24)$ & 0 & 0 \\
Control group $(\mathrm{n}=24)$ & 2 & 1 \\
$\mathrm{X}^{2}$ & 2.0 & 2.0 \\
$\mathrm{P}$ & $>0.05$ & $>0.05$ \\
\hline
\end{tabular}




\section{Discussion}

In China, the incidence and mortality of colorectal cancer are located at the third and fourth of the malignant tumors, respectively, and the incidence of colorectal cancer in China is increasing at an annual rate of twice the world average. High-risk groups of colon cancer include:

1) Family history of intestinal cancer, 2) The family has a tumor gene in patients 3) Colon polyps patients 4) Older people 5) Chronic ulcerative colitis patients 6) People with other malignant history Laparoscopic radical resection of colon cancer can remove the visible lesions, but some patients still relapse or metastasis after surgery, how to improve the risk of stage II colon cancer patients with clinical efficacy has become an important issue.

For the treatment of colonic tumors, in addition to surgery-based treatment, there are surgical treatment combined with chemotherapy, radiation therapy, targeted therapy, interventional therapy and immunotherapy and other comprehensive treatment model (MDT). For patients with high-risk stage II colon cancer after adjuvant chemotherapy can improve the radical resection rate of colon cancer, reduce the local recurrence of an important method. Stage II colon cancer risk factors include: the degree of tumor cell differentiation, tumor staging, preoperative perforation, obstruction, nerve invasion and vascular invasion, preoperative tumor marker level, margin close, uncertain or positive, etc. [1] [2]. Mumounas and other integrated NSABP C-01 C-02 C-03 C-04 four experimental analysis of stage II (1567 cases) of patients with colon cancer 5-FU/LV adjuvant chemotherapy group 5-year survival rate and mortality decreased, that II Patients with colon cancer can benefit from adjuvant chemotherapy [3].

1982 Frei first proposed the concept of neoadjuvant chemotherapy, neoadjuvant chemotherapy is mainly refers to the clinical manifestations of locally advanced lesions, in the implementation of local treatment (surgery or radiotherapy) before the start of systemic chemotherapy, because of its comprehensive treatment of tumor and local treatment Before the application, also known as the previous chemotherapy, are adjuvant chemotherapy. At present it has become an important part of the comprehensive treatment of certain tumors (breast cancer, gastrointestinal cancer). The role of neoadjuvant chemotherapy and the advantages are: 1 . To reduce the size of primary tumor lesions or metastases, reduce tumor staging, reduce tumor and surrounding tissue adhesion, control or eliminate micro-metastases, the tumor surgical resection easier, the positive rate of tumor margins decreased, part Tumor can achieve complete pathological remission, thereby improving radical resection of the tumor. 2. Once the diagnosis is clear, you can use chemotherapy drugs to control tumor growth, by judging the efficacy of neoadjuvant chemotherapy chemotherapy drugs can help to screen chemotherapy drugs, if the patient is not sensitive to preoperative chemotherapy can change the chemotherapy regimen. 3. Preoperative chemotherapy can make cancer cells in a low value-added situation, for the surgical process to reduce the iatrogenic planting [4]. 
Like Li et al. believe that neoadjuvant chemotherapy within two weeks after the chance of anastomotic fistula, three weeks after the end of chemotherapy more safe operation [5]. Therefore, for the sake of conservatism, this study is recommended for patients with neoadjuvant chemotherapy after about four weeks of surgical treatment, the same, adjuvant chemotherapy group, is also around four weeks after adjuvant chemotherapy. On the best timing of adjuvant chemotherapy, the United Kingdom SAFFA test, including a number of studies have shown that 8 weeks after adjuvant chemotherapy in patients with colorectal cancer survival rate was no significant difference with the control group, postoperative adjuvant chemotherapy later, its survival The worse the condition, and chemotherapy time for each extension of 4 weeks, OS decreased by $14 \%$. The time to start chemotherapy is affected by preoperative albumin levels, lymphatic metastases, and laparoscopic surgery [6].

In this study, we investigated the effect of neoadjuvant chemotherapy on highrisk stage II colorectal cancer:

1) By comparing the intraoperative and postoperative complications of the two groups, $\mathrm{P}$ value was greater than 0.05 , can be obtained in the experimental group postoperative complications No significant increase, is relatively safe.

2) Compared with the two groups of toxic and side effects, after SPASS software analysis, obtained $\mathrm{P}>0.05$, the two groups were not statistically significant, the experimental group and the control group did not find significant differences. Visible neoadjuvant chemotherapy is a safer, patient tolerable treatment regimen.

3) through the abdomen of the abdomen CT and preoperative review of abdominal CT were compared, after 3 - 4 courses of preoperative chemotherapy, $\mathrm{CR}$ (complete remission) and $\mathrm{PD}$ (disease progression) patients were 0 cases, $\mathrm{PR}$ (partial remission) was 11 cases $(45.8 \%$ ) and NC/SD (no change) were 13 cases (54.2\%). It was found that preoperative chemotherapy had significant effect on some patients.

4) The quality of life in the experimental group was significantly higher than that in the control group $(\mathrm{P}<0.05)$. The quality of life in the experimental group was significantly higher than that in the control group $(\mathrm{P}<0.05)$. The quality of life was significantly higher than that of the control group Patients benefit from neoadjuvant chemotherapy. Neoadjuvant chemotherapy improves clinical efficacy.

5) The survival rate of the two groups was $100 \%(24 / 24)$, the 1-year survival rate was $100 \%(24 / 24)$, the 3 -year survival rate was $92 \%(22 / 24)$. The 5 -year survival rate was $75 \%(9 / 12)$. It was found that the survival rate of patients with high-risk stage II colon cancer was significantly improved.

6) There were no recurrence or metastasis cases in the neoadjuvant chemotherapy group, and 2 cases of recurrence and 1 case of metastasis in the adjuvant chemotherapy group were compared with the recurrence or metastasis after tumor operation. The chi-square test, no statistical significance, may be due to too 
little sample, no obvious conclusion. However, we often see that many high-risk patients have no recurrence, and some low-risk patients have relapse and metastasis [7]. The overall efficacy of neoadjuvant chemotherapy in the complications of surgery and chemotherapy complications of the two no significant differences, but in patients with postoperative quality of life, the overall survival time was significantly higher and postoperative recurrence and metastasis was significantly lower, Preoperative chemotherapy can reduce tumor volume. Xia Guobing and other studies show that neoadjuvant chemotherapy can reduce the degree of tumor burden, reduce tissue reactive edema. A large number of clinical studies have shown that preoperative chemotherapy can reduce local recurrence rate and improve survival. [8] shows that the results obtained in this paper are basically the same.

Xiangcai Guo, et al. show that the simple use of clinical features to determine whether the stage II colon cancer adjuvant chemotherapy lack of evidence [9], Some patients with low risk of recurrence, and some high-risk patients without recurrence, Some patients with low risk of recurrence, and some high-risk patients without recurrence.

This article also has more defects, such as less sample size, can not evaluate the impact of chemotherapy on surgery, adjuvant chemotherapy evidence needs to be further improved and so on.

\section{Conclusion}

In summary, neoadjuvant chemotherapy plays a role in patients with high-risk second-stage colon cancer, and the experimental group benefited significantly, especially in quality of life and survival time. At the same time, neoadjuvant chemotherapy is also a safe treatment, so as to play a certain clinical guidance.

\section{References}

[1] Yan Cutsem, E., Cervantes, A., Nordlinger, B., et al. (2014) Metastatic Colorectal Cancer: ESMO Clinical Practice Guidelines for Diagnosis Treatment and Follow-Up. Annals of Oncology, 25, iii1-iii9. https://doi.org/10.1093/annonc/mdu260

[2] Benson, A.B., Schrag, D., Somerfield, M.R., et al. (2004) American Society of Clinical Oncology Recommendations on Adjuvant Chemotherapy for Stage II Colon Cancer. Journal of Clinical Oncology, 22, 3408-3419. https://doi.org/10.1200/JCO.2004.05.063

[3] Mamounas, E., Rockette, H., Jones, J., Rtalwie, S. and Wolmark, N. (1996) Comparative Efficacy of Adjuvant Chemotherapy in Patients with Ducks B versus Ducks C Colon Cancer Results from Four NSABP Adjuvant Studies (c-01,c-02,c-03,c-04). Proceedings of American Society of Clinical Oncology, Abstract 461.

[4] Qiao, T.Y. and Wang, X.S. (2012) Advances in Diagnosis and Treatment of Liver Metastasis of Colorectal Cancer. Chinese Cancer Clinical and Rehabilitation, 19, 572-575.

[5] Li, L.K. and Tian, Z.G. (2011) Effect of Neoadjuvant Chemotherapy on Surgical Anastomosis in Colon Cancer. Contemporary Medicine, 9, 45-46.

[6] Dong, S.J., Han, W.W., et al. (2015) Analysis of Factors Influencing Independent 
Factors of Adjuvant Chemotherapy Delay after Resection of Colorectal Cancer. Chinese Journal of Experimental Surgery, 32, 1421-1423.

[7] Benson, A.B. and Hamilton, S.R. (2011) Path toward Prognostication and Prediction: An Evolving Matrix. Journal of Clinical Oncology, 29, 4599-4601. https://doi.org/10.1200/JCO.2011.37.8646

[8] Xia, G.B. and Cao, Y.G. (2011) Analysis of Curative Effect of Neoadjuvant Chemotherapy for Colon Cancer in 18 Cases. Chinese Journal of Experimental Surgery, 28, 818-818.

[9] Cai, G.X., Dai, W.X. and Cai, S.J. (2016) Current Situation and Future of Multidisciplinary Treatment of Colorectal. Chinese Journal of Gastrointestinal Surgery, 19, 607-610. 